\title{
Los ácidos grasos omega-3 no reducirían la mortalidad, la enfermedad cardiovascular ni el cáncer
}

Hooper L y col. BMJ 2006; 332(7544):752-60

\section{Objetivo}

Evaluar el efecto de los ácidos grasos omega-3 de cadena corta y larga sobre la mortalidad total, eventos cardiovasculares y cáncer.

\section{Fuente y selección de datos}

Se buscaron ensayos controlados aleatorizados (ECA) en base de datos electrónicas Cochrane Library, Medline, Embase, National Research Register y SIGLE hasta febrero de 2002 en los cuales se hubiesen administrado omega-3 por un período igual o mayor a 6 meses, en adultos (con o sin factores de riesgo cardiovascular). También fueron incluidos aquellos estudios de cohorte en donde se evaluó la ingesta de omega-3 relacionándolo con resultados clínicos, al menos a los 6 meses.

\section{Resultados}

De los 15.159 títulos y resúmenes evaluados se analizaron 48 ECA (con 36.913 participantes) y 41 estudios de cohorte. Los resultados de los estudios clínicos fueron inconsistentes. La valoración no mostró una fuerte evidencia en cuanto a la reducción del riesgo de la mortalidad total o eventos cardiovasculares combinados (Tabla 1) en los participantes que tomaron ácidos grasos omega-3 suplementarios. Analizando sólo los estudios con menor sesgo*, aunque más consistentes, tampoco llegaron a demostrar que el omega-3 reduzca la mortalidad total (RR 0,98 [IC95\% 0,70-1,36]) ni de even- tos cardiovasculares $(1,09[0,87-1,37])$. Los estudios de cohorte sugirieron reducción de mortalidad $(0,65[0,48-0,88]$. No queda claro si hubo un adecuado ajuste* por confundidores*. En el subgrupo de estudios de omega-3 de cadena larga, tampoco es clara la reducción de la mortalidad total $(0,86[0,70-1,04])$ ni de eventos cardiovasculares $(0,93[0,79-1,11])$. El riesgo de cáncer no se vio incrementado ni en los ECA (Tabla 1) ni en los estudios de cohorte $(1,02[0,87-1,19)$, pero no pueden excluirse daños clínicos importantes.

Tabla 1. Resultados del meta-análisis de ECA

\begin{tabular}{|c|c|c|c|c|c|}
\hline Resultados & $\begin{array}{l}\text { Omega } 3 \text { Alto } \\
\text { nNI (\%) }\end{array}$ & $\begin{array}{c}\text { Omega } 3 \text { Bajo } \\
n / N(\%)\end{array}$ & $\begin{array}{c}\mathrm{RR} \\
\text { (IC } 95 \%)\end{array}$ & p & $\mathbf{P}^{2}$ \\
\hline Mortalidaa total & $960 / 16.701(5,7)$ & $1035 / 16.492(6,3)$ & $0,87(0,73-1,03)$ & NS & $42 \%$ \\
\hline Eventios cardiovasculares & $133446.979(7,9)$ & $1224 / 16.646(7,8)$ & $0,95(0,82-1,12)$ & NS & $65 \% \%^{*}$ \\
\hline Cáner & $21118.752(24)$ & $18886681(2,2)$ & $1,07(0,88-1,30)$ & NS & $0 \%$ \\
\hline
\end{tabular}

*Un I2 $>60 \%$ indica significativa heterogeneidad* pero esta bajaba a $48 \%$ en el análisis de sensibilidad por calidad.

\section{Conclusión}

Los ácidos grasos omega-3 de cadena corta y larga no tienen un efecto claro sobre la mortalidad total, eventos cardiovasculares combinados o cáncer.

\section{Comentario}

El tratamiento de las hiperlipidemias tiene como objetivo reducir la morbimortalidad por enfermedad cardiovascular, así como la mortalidad global. El cambio de los hábitos alimentarios, reduciendo el consumo de grasas saturadas, es una de las indicaciones que habitualmente utilizamos a estos fines. El consumo de ácidos grasos omega-3 podría proteger contra la enfermedad cardiovascular mediante el descenso de la presión arterial y de la frecuencia cardiaca, reduciendo los niveles séricos de triglicéridos, la tendencia trombótica, la inflamación y las arritmias; mejorando la función endotelial, la sensibilidad a la insulina y la estabilidad plaquetaria'. También tenemos que tener en cuenta que en los aceites de pescado y en los pescados grasosos se han encontrado componentes tóxicos como el metilmercurio, dioxinas y bifeniles policlorinados que ocasionan daño luego de mucho tiempo de ser utilizados. El primero podría aumentar el riesgo de infarto de miocardio y causar daño neurológico, mientras que los últimos aumentarían el riesgo de cáncer. De hecho el estudio de Burr y col (de mayor seguimiento y segundo en cantidad de eventos) ${ }^{2}$ demostró efectos perjudiciales a diferencia de los otros ECA.

Una revisión previa' ${ }^{1}$ sobre suplemento de omega-3 en enfermedad cardiovascular que incluyó 11 de los 48 ECA de la actual revisión (entre los que no estaba el estudio de Burr ${ }^{2}$ pero sí dos de los excluidos de la actual revisión por ser intervenciones multifactoriales), mostró reducción de la mortalidad en términos generales. Tanto es así que guías inglesas ${ }^{3}$ recomiendan a la población general consumir más aceite de pescado y mayores cantidades sobre todo a aquellas personas que tuvieron un infarto de miocardio. Sin embargo, esta nueva revisión más completa y metodológicamente robusta, no muestra evidencia de un efecto beneficioso del consumo de omega-3 sobre la mortalidad, eventos cardiovasculares, cáncer y accidente cerebrovascular. Por lo tanto, para entender los efectos de los ácidos grasos omega-3 sobre la salud necesitamos más ECA de alta calidad y de mayor duración que permitan evaluar mejor los riesgos y beneficios sobre estas patologías.

\section{Conclusión del comentador}

En base a los resultados poco consistentes en cuanto a las propiedades de los ácidos grasos omega-3 presentados en esta revisión sistemática, lo ideal sería contar con más ECA de mayor calidad y duración a fin de disponer de datos complementarios que clarifiquen evidencia actualmente disponible.

Marcela Micha [ Médica de Familia. Hospital Italiano de Buenos Aires. ]

Micha M. Los ácidos grasos omega-3 no reducirían la mortalidad, la enfermedad cardiovascular ni el cáncer. Evid. Actual. Práct. Ambul. 9(4);106. Jul-Ag. 2006. Comentado de: Risk and benefits of omega-3 fats for mortality, cardiovascular disease, and cancer: systematic review. Hooper L, Thompson R, Harrison R y col. BMJ 2006;332(7544):752-60. PMID: 16565093

\section{Referencias}

1. Bucher HC, Hengstler P, Schindler C y col. N3 polyunsaturated fatty acids in coronary heart disease: a meta-analysis of randomized controlled trials. Am.J Med 2002;112:298-304

2. Burr ML, Ashfield-Watt PA, Dunstan FD, Fehily AM, Breay P, Ashton T, et al. Lack of benefit of dietary advice to men with angina: results of a controlled trial. Eur J Clin Nutr 2003;57:193-200.

3. Wood D, Durrington P, Poulter N y col. Joint British recommendations on prevention of coronary heart disease in clinical practice. Heart 1998;80:S1-29 